\title{
Influence of shells on mating behavior in the hermit crab Calcinus tibicen
}

\author{
B.A. Hazlett and L.C. Baron \\ Department of Biology, University of Michigan, Ann Arbor, MI 48109, USA \\ Received December 21, 1987 / Accepted October 9, 1988
}

Summary. Populations of the intertidal hermit crab Calcinus tibicen were observed in the laboratory and reproductive behaviors recorded. Of the 218 interactions, 68 resulted in copulation(s). Male and female sizes were positively correlated. Male size affected copulation success in a non-linear fashion. In particular, the largest males did not obtain any copulations. This was largely a consequence of the shell species occupied by large individuals; males in Nerita sp and Cittarium pica shells were unsuccessful in courtship. The ability to execute precopulatory rotation of the female was negatively affected by certain shell types. Repeated pairings of individuals suggested some level of individual recognition within the reproductively active population.

\section{Introduction}

The mating behavior of many animals is influenced by resources. For example, in the case of a number of species, aspects of the territory that males hold influence the chances of a male obtaining matings (Halliday 1978; Arnold 1983; Arcese 1987; Borgia et al. 1987; Christy 1987). Male-male competition for desirable territories is usually predicated upon the eventual influence that possession of those resources will have upon reproduction.

The gastropod shells inhabited by hermit crabs represent an unusual resource in several ways. Once they have metamorphosed to the crab stage, individual hermit crabs must inhabit a shell (or other object) for protection against predation and environmental stresses (Hazlett 1981). Inhabitation of the correct size and species of shell has positive effects on the degree of predator protec-

Offprint requests to: B.A. Hazlett tion (Vance 1972; Bertness 1981a), the rate of growth (Markham 1968; Bertness 1981 b; Fotheringham 1976) and the fecundity of females (Fotheringham 1980; Bertness 1981 b). As crabs grow, once-optimal shells become less advantageous and larger shells must be found. This can involve a shift to a different species of shell with associated changes in the shape of the shell. Crabs can obtain new shells by several means but the two primary methods of obtaining new resources are by finding recently emptied shells (McLean 1974; Rittschof 1980 ) or by exchanging shells with other crabs (Hazlett 1966, 1984; Imafuku 1983).

The influence of shell type and size on the reproductive output of female hermit crabs has been documented for several species. Occupation of lessdesirable species of shells can have strong influences on the probability of a female having any eggs on her pleopods (Bach et al. 1976). The relative size of a shell can negatively influence fecundity both if the.shell is too large and if it is too small (Bertness 1981 b). The purpose of this study was to investigate the influence of shell type and size on male reproductive success. We studied the influence of the shells that males occupy on the probability of their obtaining matings in the intertidal diogenid hermit crab Calcinus tibicen. The mating behavior of this species has been described earlier (Hazlett 1966).

\section{Materials and methods}

The observations were done at the Discovery Bay Marine Laboratory, Discovery Bay, Jamaica during February and March, 1987. Individuals of the diogenid hermit crab Calcinus tibicen were collected from several locations near the Marine Laboratory and placed in a concrete water table in the laboratory. The water table was 45 by $91 \mathrm{~cm}$ wide with a water depth of about $6 \mathrm{~cm}$ and was continually supplied with new sea water. Between 200 and 250 individuals were maintained in the water table during observation periods. Observation of the crabs was 
continuous most days from $0800 \mathrm{~h}$ to about $1600 \mathrm{~h}$. The occurrence of shell exchange behaviors was recorded for another study (Hazlett 1989). All individuals were placed individually in compartments of plastic trays whenever observations were not in progress, thus no social interactions (reproductive attempts or shell exchanges) could occur unobserved. Whenever crabs took part in any reproductive behaviors, they were briefly removed (after the interaction had been completed) and a number placed on the back of the animal's gastropod shell. After about a week of continued observations, marked individuals were removed for measurements and new individuals added to the water table.

All individuals that participated in interactions had the following parameters recorded: shell species occupied, crab sex, crab size and shell size. Crab size was characterized by cephalothorax length (=cl) and "dry" weight after the crab was blotted dry on a paper towel. The shell measurements included total length, shell dry weight and shell internal volume. Volume was estimated by filling the dry empty shell with fine sand and weighing it. By subtraction and consideration of the specific density of the sand, the volume of each shell could be calculated.

The sizes of shells that crabs occupied were compared with the size of shell each crab would choose if given a free choice among shells of that species. Desired shell sizes were determined by a series of free-access experiments in which about 70 adult C. tibicen were placed with about $200 \mathrm{empty}$ gastropod shells of one species and crab and shell parameters measured after $48 \mathrm{~h}$. These data allowed calculation of a regression equation relating crab size and shell size for a number of shell species.

The behavior patterns recorded in this study have been described elsewhere (Hazlett 1966). The pre-copulatory behavior of most diogenid hermit crabs, including Calcinus tibicen, involves a series of movements by male crabs while holding a female crab's shell with their ambulatory legs. All reproductive behavior patterns occur with the pair in an opposed position (Hazlett 1966), shell apertures facing one another. While the male balances himself with one of the second pair of ambulatory legs, he rotates the female's shell back and forth about an axis perpendicular to and through the plane of the female shell aperture. These rotations by the male are the most prevalent pattern executed by male C. tibicen. The stroking movements of the female by the male's chelipeds which are more common in some species of Calcinus (Hazlett 1972) are infrequent in this species. After some bouts of rotation, female $C$. $t i$ bicen may stroke the mouth parts of the male with her ambulatory legs and chelipeds and the pair eases partially out of their gastropod shells and copulate.

The behavior pattern of shell rotation was used as the indicator that a reproductive interaction was occurring. When they are attempting a shell exchange, crabs also get in the opposed position, thus that posture can not be used to discriminate between shell resource contests and reproductive behavior. Rotations always occur before copulation in this species and whenever rotations have been observed they have been executed by a male to a female. Whenever a pair of animals was observed in the opposed position, the time was noted and observations were begun. Observations continued only if rotations were seen. The number of bouts of rotation were recorded and one of three outcomes followed. If another crab tried to interrupt the pair, this was recorded. If the interruption attempt was successful, that ended the interaction between the first pair. The second crab was considered a courting male only if he was observed to execute rotation behavior. The second type of outcome of any pair was that the rotating male just released his grasp of the female's gastropod shell and walked away after some number of rotations.
The third type of outcome was copulation. This was recorded whenever both the male and female eased out of their shells far enough that the genital openings were brought very near each other and the male contracted his body such that a sharp angle between his cephalothorax and abdomen was made. It is during such a contraction that the sexual appendages of the male could be seen moving back and forth between the male and female, apparently transferring spermatophores (Hazlett 1966). If only the male eased out of his shell exposing most of his cephalothorax, no copulation was recorded. Copulation lasted between several seconds and almost a minute in a few cases. The time of copulation was recorded and the pair observed until they separated. Any further rotations by the male were recorded. The animals were left in the water table and could engage in additional interactions until observations ceased for that day.

At the end of each day of observation, females that had been involved in interactions that day or during recent days were placed in individual small beakers overnight so that the release of larvae could be detected. After about a week of additional observation and monitoring, females were removed from their shells and size measurements taken. Any shell exchanges between marked individuals were recorded and the identification of crabs adjusted accordingly.

Once a week, a census of all the shell species represented in the water table was taken in order to characterize the array of shells occupied by the individuals of $C$.tibicen being observed. The sex of the occupants of those shells was not known unless they were marked individuals. Shell exchange interactions (Hazlett 1989) were monitored and the 148 shell-related interactions observed yielded data on the shell species occupied by non-initiating males in particular.

As female crabs were measured, if one had eggs on her pleopods, they were removed and the number of the eggs counted. These counts were used to characterize the relationship between female size and the number of eggs carried.

Data were analyzed by chi-square, linear regression, and ANOVAs. Statistical tests were carried out on numbers in categories and not on percentages or ratios, which are presented for illustrative purposes only.

\section{Results}

A total of 218 interactions were observed which involved rotation of a female by a male (104 different males). Of these, 68 (involving 37 different males) resulted in one or more copulations. Seven of the 68 cases involved more than one copulation (range 2-5) between the same pair without any period of separation. These cases were counted as single instances of copulatory behavior in the analyses, thus the males involved were counted only once. Females that copulated often released mature larvae that night or during the next couple of nights. A number of females copulated on two or three successive days and a few copulated on four successive days. The majority of copulations involved a female that was berried with very mature larvae. Females rarely molted after copulation, at least at this time of year (February-March), and in some cases the female was followed long 


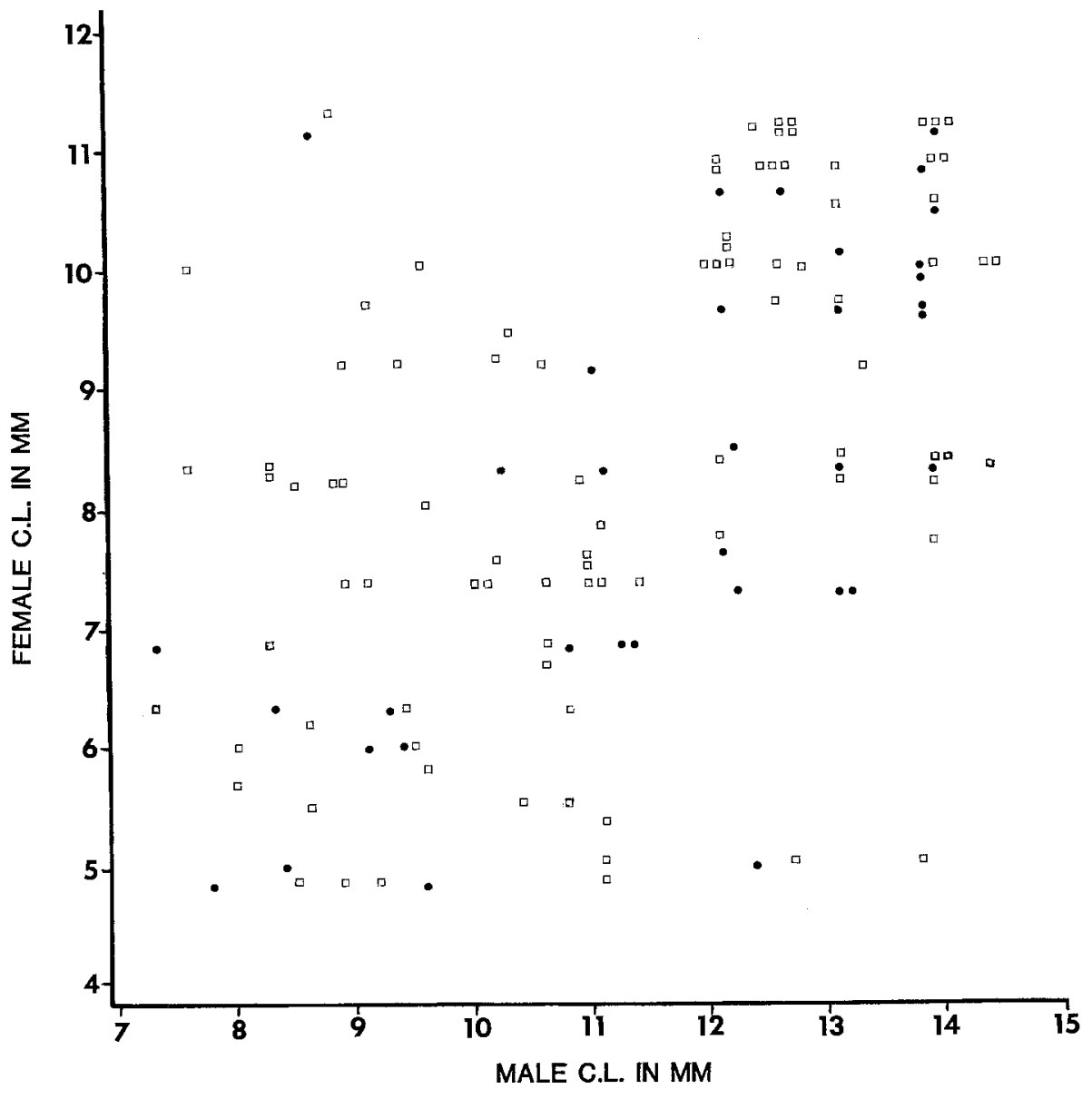

Fig. 1. Relationship between male and female size (mm cephalothorax length) in pairs of Calcinus tibicen. Open squares indicate interactions which did not end in copulation, the closed circles interactions that did involve copulation enough that she brought out a new batch of eggs following the copulation.

The number of reproductive interactions per day peaked around February 17-18 and was very low around February 23 to March 3 . Unfortunately the latter days coincided with a period of interrupted water flow at the Marine Laboratory and it is not possible to determine if the change in the level of mating activity represents a rhythm.

In the 68 cases that involved copulation, males held females for an average of 14.3 minutes (range 0.5 to 111) and executed an average of 7.1 bouts of rotation (range 1 to 46). The averages for the cases which ended without a copulation $(n=150)$ were 13.0 minutes and 6.4 bouts of rotation (neither measure significantly different comparing interactions ending with a copulation and those without copulations). In the case of the pairs that did copulate, males continued to hold the female in the opposed position for an average of $12 \mathrm{~min}$ utes after copulation and executed about one additional bout of rotation $($ mean $=0.89)$. There was no relationship between either of these post-copulatory behavioral measures and crab size (male or female) ( $P>0.10$ for all regressions) or the species of shells inhabited ( $P>0.10$ for both ANOVAs). Individual females copulated twice on the average with a maximum of ten copulations for one female. There was no relationship ( $P>0.10$ for all analyses) between the number of prior copulations a female had and any measures of crab size (male or female), the shell species occupied by either animal, or male behavior patterns (minutes of precopulatory holding, number of bouts of rotation, rate of bouts of rotation). It was particularily interesting that prior reproductive behavior had no effect upon the female's responses to male precopulatory behavior patterns.

\section{Size effects}

There was a significant correlation between male and female sizes. Crab weights in pairs that copulated were positively related to one another $(r=$ 0.393 for weight, 0.487 for cephalothorax lengths, both $P<0.001$ ) although there was considerable unexplained variance (Fig. 1). This was true both for pairs that copulated $(r=0.55$ for cephalothorax 
Table 1. Size classes ( $\mathrm{mm}$ cephalothorax length) frequencies for male $C$. tibicen involved in various categories of social behavior. The success rate was calculated by dividing the number of copulations in that size class by the total number of reproductive events involving that size class. The Reproductive Activity Ratio was obtained by dividing the percentage of the total reproductive behavior cases involving males in that size class by the percentage of males in that size class. The Copulation Ratio was obtained by dividing the percentage of the copulations obtained by males in a size class by the percentage of males in that size class. The shell exchange data refer to male non-initiators only

\begin{tabular}{|c|c|c|c|c|c|c|c|c|c|}
\hline \multirow[t]{2}{*}{ Sizes } & \multicolumn{2}{|c|}{ Shell exchanges } & \multicolumn{2}{|c|}{$\begin{array}{l}\text { All cases } \\
\text { reproductive behavior }\end{array}$} & \multicolumn{2}{|c|}{ Copulations } & \multirow{2}{*}{$\begin{array}{l}\text { Success rate } \\
{[\%]}\end{array}$} & \multirow[t]{2}{*}{$\begin{array}{l}\text { Repo activity } \\
\text { ratio }\end{array}$} & \multirow[t]{2}{*}{$\begin{array}{l}\text { Copulation } \\
\text { ratio }\end{array}$} \\
\hline & $n$ & {$[\%]$} & $n$ & {$[\%]$} & $n$ & {$[\%]$} & & & \\
\hline $5.5-6.4$ & 4 & 3.2 & 0 & 0 & 0 & 0 & - & 0 & 0 \\
\hline $6.5-7.4$ & 7 & 5.7 & 2 & 1.1 & 1 & 1.9 & 50 & 0.2 & 0.3 \\
\hline $7.5-8.4$ & 10 & 8.1 & 9 & 5.0 & 4 & 7.8 & 44 & 0.6 & 1.0 \\
\hline $8.5-9.4$ & 15 & 12.2 & 29 & 16.2 & 7 & 13.7 & 24 & 1.3 & 1.1 \\
\hline $9.5-10.4$ & 29 & 23.6 & 18 & 10.1 & 8 & 15.7 & 44 & 0.4 & 0.7 \\
\hline $10.5-11.4$ & 23 & 18.7 & 19 & 10.6 & 3 & 5.9 & 16 & 0.5 & 0.3 \\
\hline $11.5-12.4$ & 14 & 11.4 & 20 & 11.2 & 4 & 7.8 & 20 & 1.0 & 0.6 \\
\hline $12.5-13.4$ & 8 & 6.5 & 34 & 19.1 & 9 & 17.6 & 26 & 2.9 & 2.7 \\
\hline $13.5-14.4$ & 9 & 7.3 & 40 & 22.5 & 14 & 27.4 & 35 & 3.8 & 3.7 \\
\hline $14.5+$ & 4 & 3.2 & 7 & 3.9 & 1 & 1.9 & 14 & 1.2 & 0.6 \\
\hline Totals & 123 & 100.0 & 178 & 100.0 & 51 & 100.0 & & 1.0 & 1.0 \\
\hline $\begin{array}{l}\text { or } \\
\text { mean }\end{array}$ & & & & & & & 29 & & \\
\hline
\end{tabular}

lengths) and pairs that did not copulate $(r=0.47)$. The fact that larger males courted and copulated with larger females is not surprising and means that larger males had the potential to fertilize more eggs since there was a strong relationship between female weight and number of eggs $(r=0.765, P<$ 0.001 ). It is interesting that in the cases where the male simply walked away from the female after at least one rotation but no copulation occurred, the correlation between male and female size was not significant $(r=0.260, P=0.155)$.

The males that were involved in courtship were larger $($ mean $=11.1 \mathrm{~mm} \mathrm{cl})$ than the males involved in shell exchange behavior (mean $=9.5 \mathrm{~mm})(t=$ $6.18, d f=287, P<0.01)$. There was no difference in the sizes of females (mean $=8.3 \mathrm{~mm}$ for courted females, $8.1 \mathrm{~mm}$ for females involved in shell exchanges as either initiator or non-initiator). There was a large range of male sizes among the individuals that successfully courted and copulated and overall there was no difference in the size of successful males $(11.0 \mathrm{~mm} \mathrm{cl})$ and unsuccessful males $(11.2 \mathrm{~mm} \mathrm{cl})(t=0.57, d f=175, P>0.05)$.

The distribution of copulations among size classes of males was significantly different from that predicted by the frequency of those size classes in the general population (Table 1) $\left(\chi^{2}=41.1, d f=\right.$ $9, P<0.01)$. The smaller size males in the water table were not able to court successfully, at least in part because of the frequency of interruption by larger males. Both the medium-small and the medium-large size male individuals of $C$.tibicen obtained a disproportionate number of matings, given their frequency in the male population in the water table. The relationship between male size and both mating activity and mating success does not appear to be a simple function. That is, there is not a gradual increase in probability of matings. Medium sized males (about 7.0 to $9.0 \mathrm{~mm} \mathrm{cl}$ ) seemed to be reasonably successful in obtaining matings (see Table 1). However slightly larger males (10.0 to $11.5 \mathrm{~mm} \mathrm{cl}$ ) were less successful and were not involved in reproductive activities as frequently as one would predict from their representation in the population (calculated from the shell exchange data on nopn-initiators). Medium-large males $(12.0-13.5 \mathrm{~mm} \mathrm{cl})$ were both reproductively active and successful.

However, the very largest males (over $14.0 \mathrm{~mm} \mathrm{cl}, n=7$ individuals) were unsuccessful in obtaining matings. These males were active in interrupting matings and the mean size of interrupting males was significantly larger than the mean size of successful copulators (mean $12.0 \mathrm{~mm}$ cl vs. $11.0 \mathrm{~mm} \mathrm{cl}, t$-test $=2.15, d f=68, P<0.05)$. The very largest male $(16.7 \mathrm{~mm} \mathrm{cl})$ repeatedly interrupted pairs and courted females but was unsuccessful at obtaining copulations. This was not a result of quantitative differences in vigor of courtship since there was no decline in the rate of rotations (bouts of rotation/minute of courtship) or the number of minutes of courtship with increased 
Table 2. Frequencies of shells occupied by males in various categories of reproductive behavior compared to shell frequencies in the laboratory population. All numbers except sample sizes are percentages

\begin{tabular}{|c|c|c|c|c|c|}
\hline Shell sp. & Water table count & $\begin{array}{l}\text { Males in shell } \\
\text { exchange as non-initiators }\end{array}$ & $\begin{array}{l}\text { Males involved } \\
\text { in courtship }{ }^{a}\end{array}$ & $\begin{array}{l}\text { Interrupted } \\
\text { courtships/attempts }\end{array}$ & $\begin{array}{l}\text { Successful } \\
\text { copulations }\end{array}$ \\
\hline Leucozonia & 34.0 & 28.4 & 43.6 & 41.0 & 48.5 \\
\hline Cerithium & 27.1 & 3.2 & 0 & - & 0 \\
\hline Cittarium & 4.8 & 24.4 & 12.8 & 21.0 & 11.7 \\
\hline Nerita & 7.8 & 9.7 & 4.6 & 30.0 & 0 \\
\hline Thais & 4.8 & 11.4 & 19.7 & 37.2 & 23.5 \\
\hline Cymatium & 5.5 & 7.3 & 13.8 & 33.3 & 14.7 \\
\hline Astrea & 2.0 & 4.1 & 1.8 & 100.0 & 0 \\
\hline Tegula & 5.3 & 0.8 & 1.3 & 67.0 & 1.5 \\
\hline Fasciolaria & 0.5 & 0.8 & 1.8 & 75.0 & 0 \\
\hline Other species & 5.4 & 3.2 & 0 & - & 0 \\
\hline$n=$ & 829 & 123 & 218 & 81 & 68 \\
\hline
\end{tabular}

a Counting both successful and unsuccessful courtship attempts

b $\%$ of the courtship attempts by males in that species which were interrupted

size (e.g. $r=-0.10$ between rate of rotation and male weight, $P>0.10$; similar values for all relationships measured). The size effects on mating success at least for the larger males may be explained by consideration of the shell species occupied.

\section{Shell species effects}

The shell species that a male crab occupied had a very strong effect on its probability of obtaining a mating. This effect seems to result from at least three factors: (1) males in some species of shell never engaged in any copulatory activity, (2) males in some shells tried but were never successful due to ease of interruption, and (3) males in some species of shells tried but females did not respond to male rotations.

As shown in Table 2, if we use either the shell counts of the whole population in the water table (sex of crabs unknown) or the distribution of shell types among males that were non-initiators in shell exchange activity as a base line of frequency of male-inhabited shells, males in some species of shell do not try to court females. Males in Cerithium shells and Nerita are clearly under-represented in the category "involved in courtship". Males in some species of shell were sexually active but under-represented in the successful copulators category $\left(\chi^{2}=40.9, \quad d f=9, \quad P<0.01, \quad\right.$ comparing number of successful copulators by shell species occupied and the number expected based upon the frequencies of shell species occupation from the shell exchange data). This was especially true for males in Cittarium pica and in Nerita sp shells. Males Leucozonia shells were successful at a rate predicted from their frequency in the reproducti- vely active male population while males in Thais deltoidea and Cymatium were more successful than one would predict. Males in less common species of shell were uniformly unsuccessful in obtaining matings.

These differences in mating success were not a result of any difference in courtship vigor among the males in shell species that did attempt rotations. There were no differences among males in different shell species in the number of bouts of rotation, the rate of rotations or the number of minutes courted (analyses of male shell species occupied and the above three behavior measures yielded ANOVA F-values associated with $P>0.10$ for both successful and unsuccesful courtship attempts). Males in Cittarium shells (10 different males) courted just as long and as vigorously as did males in Leucozonia, Thais, or Cymatium shells (46, 14, and 12 individuals respectively) but females did not respond to their precopulatory behaviors as quickly. That is, after the same number of minutes of courtship or the same number of rotations of the female, a male in a Thais or Leucozonia shell was more likely to obtain a mating than a male in a Cittarium or Nerita shell.

Our observations of rotation behavior suggest a possible basis for this lack of success by males in some species of shells: males in some shells are unable to properly execute courtship behavior. Particularly in the case of Nerita (zero copulations from attempts by ten individuals) and Cittarium shells (eight copulations from 20 attempts by ten different males), the round, open shape of the aperture and the angle of the collumella to the plane of the aperture seem to contribute to several problems. The ambulatory legs of the crabs appeared pinched together medially such that they had diffi- 
culty both in grasping the females' shells and in rotating them properly. Secondly, males in those shells tended to fall over more frequently while trying to rotate females. The extreme case was a very large male (13.8 $\mathrm{mm} \mathrm{cl}, 1.55$ grams weight) in a Cittarium shell that did 'successfully' court a female. The female had eased partially out of her shell and the male was easing out of his shell. Just as copulation should have occurred, the male lost his balance and fell forward out of his shell completely. This caused the female to pull back into her shell partially and she walked away as the shellless male was trying to get back into his shell. The more ovoid, elongate aperture of shells such as Thais or Leucozonia appeared to allow easier balancing by males and permit execution of rotations more readily. The difficulty of balancing by males in Cittarium and Nerita resulted in easier interruption on those males by other males and the inability to rotate females properly appeared to result in females not being stimulated to copulate as readily.

While the type of shell a male occupied had significant effects on his probability of obtaining a mating, the degree of shell fit did not. Sufficient data were available to compare the shell fit of successful and unsuccessful courting males in three species of shell (Leucozonia, Thais, and Cittarium). For each male, the desired shell size in terms of internal volume was calculated from the regression equations derived from the free-access data. The deviation of each male's currently occupied shell from his desired shell size was calculated and the means compared for the two classes of males. While there was a barely significant effect in the case of Thais (successful males had a larger deficit), the results overall indicate no striking difference (Table 3).

Females crabs that were involved in reproductive behavior were in a different array of shells $\left(\chi^{2}=236, P<0.01\right)$ than female crabs involved in shell exchanges (Table 4). Individuals in Thais and Cerithium shells were more frequently engaged in reproductive behavior while females in Cittarium and Nerita were less frequently observed in reproductive behavior than would be expected, from their frequency in the shell exchange data. There was no significant difference in the array of shells occupied by females that copulated as compared to those involved in any reproductive behaviors $\left(\chi^{2}=6.1, P<0.10\right)$.

There was no obvious tendency for the male and female of a pair to be in the same species of shells other than what would be expected by the frequencies of those shells in the reproductive population.
Table 3. Mean milliliters of deviation from desired shell volume for males that successfully copulated as compared to males that did not obtain copulations

\begin{tabular}{llllll}
\hline $\begin{array}{l}\text { Shell } \\
\text { species } \\
\text { occupied }\end{array}$ & $\begin{array}{l}\text { Successful } \\
\text { copulations }\end{array}$ & $\begin{array}{l}\text { Unsuccessful } \\
\text { attempts }\end{array}$ & $\begin{array}{l}t \text { - } \\
\text { statistic }\end{array}$ & $P$ \\
\hline Leucozonia & $0.42 \mathrm{ml}$ & 0.36 & 0.90 & 46 & 0.10 \\
Cittarium & 0.51 & 0.56 & 0.23 & 24 & 0.10 \\
Thais & 1.50 & 1.10 & 2.12 & 31 & 0.05 \\
\hline
\end{tabular}

Table 4. Shells occupied by female $C$. tibicen engaged in either shell exchange attempts or in reproductive behaviors. Percentage occupation for each category of behavior

\begin{tabular}{llll}
\hline Shell species & $\begin{array}{l}\text { Shell } \\
\text { exchange } \\
\text { behavior }\end{array}$ & $\begin{array}{l}\text { All } \\
\text { reproductive } \\
\text { behaviors }\end{array}$ & Copulations \\
\hline Leucozonia & 38.0 & 34.7 & 40.3 \\
Cerithium & 10.9 & 24.9 & 26.9 \\
Cittarium & 13.0 & 0.5 & 0.0 \\
Nerita & 11.9 & 7.5 & 3.0 \\
Thais & 2.1 & 14.1 & 19.4 \\
Cymatium & 9.8 & 8.5 & 3.0 \\
Astrea & 3.2 & 0.0 & 0.0 \\
Bursa & 3.2 & 0.0 & 0.0 \\
Tegula & 3.2 & 4.2 & 3.0 \\
Turbo & 1.1 & 5.6 & 4.5 \\
Others & 3.2 & 2.3 & 1.5 \\
\hline
\end{tabular}

\section{Individual identifications}

On a number of occassions, we observed the same two individuals paired more than one times. There were 15 instances of a male rotating the same female on multiple occassions (10 twice, five cases of three pairings), either on separate days (after they had been in individual containers overnight) or on the same day but the pair physically separated from one another in the water table for at least one hour. To calculate the number of times multiple pairings would be expected by chance, the observation periods were examined in overlapping, three day periods. For each three day period, the number of individual males and individual females that we observed engaged in reproductive activities was counted. The probability of any given pair being seen was then calculated and this probability multiplied by the actual number of pairings seen over that three-day period. These values were summed for all three-day periods and this predicted that based upon chance alone, 3.45 multiple pairs would have been seen. This is significantly different than the 15 multiple pairs that were observed $\left(\chi^{2}=35.0, P<0.01\right)$. 


\section{Discussion}

The ability to obtain resources associated positively with fitness should be of advantage for animals in any resource-utilizer system. The foci of past studies of hermit crabs and their gastropod shell resources have been on aspects of (predator) protection and influences on growth (see reviews by Hazlett 1981; Scully 1983), both of which can influence individual fitness as well as population growth. The results of this study demonstrate that in addition, the shell that a crab occupies can influence fitness via sexual selection. Thus the abilities to obtain and learn to utilize new types of shell resources as a crab grows will be of advantage for a wide variety of selective reasons.

The ability of males to obtain mates is directly influenced by male size in a variety of crustacean species (Salmon 1983). The current study of hermit $\mathrm{crab}$ reproductive behavior indicates that as male size increases, up to a point, the probability of copulatory success increases for individuals of $\mathrm{Cal}$ cinus tibicen. Smaller males are limited in the size of females they are likely to mate with, thus the number of eggs they might fertilize. Male-male competition as well as other processes probably contribute to this limitation. As males grow (a process that optimal shell size inhabitation facilitates), the size of females they can mate with increases and thereby the possible number of their offspring increases. However, male-male competition (and interruption) can negatively influence a male's chance of mating, particularly if a number of large male conspecifics are in the area.

In addition to size influences, a male hermit crab's chances of mating are very strongly influenced by the type of gastropod shell he occupies. Shells with more rounded apertures (Nerita, Cittarium, Astrea) apparently make a male's precopulatory activity less effective and consequently strongly negatively influence his chances of reproducing. Such shells also appear to negatively influence female fecundity (Bach et al. 1976) in this and other diogenid species. It is not surprising that these genera of shells are not chosen by individuals of Calcinus tibicen when crabs are given a choice of shell types (Hazlett 1989).

The generality of these observations could be questioned given that all observations were done in the laboratory in a concrete water table, i.e. one type of substrate. However, Calcinus tibicen is restricted (at least in Jamaica) to old coral rocky areas which very closely approximate the surface texture conditions encountered in the laboratory. While some influence of environmental features on these data can not be ruled out, the strong effect of shell type on male reproduction would appear to be very important.

It would appear that at least two processes contribute to the reduced likelihood of mating for males in these less-desired shells. First, crabs in these shells spend more time engaged as initiators in shell exchange behavior (Hazlett 1987, 1989). Crabs (male and female) in Astrea, Nerita, and Cittarium shells initiate many more shell exchange interactions than do crabs in Thais or Leuconzonia shells. Consequently, males in the former three genera have less time to engage in reproductive behavior. Secondly, when males in such shells do execute precopulatory behavior patterns, they are less successful. The same number of rotations by a male in a Cittarium/Nerita shell is less likely to result in a female easing out of her shell partially and soliciting copulation than those rotations by a male in a Thais shell. This could be viewed as female choice by the female hermit crabs (nonresponse to "clumsy" rotations) or as inaccurate categorization of behavior patterns as appropriate precopulatory behavior by the human observers. In either event, the differential responses of females to the behavior patterns of males that occupy different species of shells markedly affects the chances of male hermit crab mating. This female choice behavior is not linked to any resource that can affect the female in any obvious manner.

Whatever the complex of factors leading to the reduced reproductive success of males in some species of shells, male hermit crabs are presented with an interesting conflict. As they grow, individual males increase their chances of mating with the most fecund females. However, for individuals of C. tibicen in Jamaica (and elsewhere in the Caribbean), the supply of large shells of the desired species is very limited. The largest, reasonably common Thais deltoidea and Leucozonia nassa shells can be occupied by males $12.5-13.5 \mathrm{~mm}$ cephalothorax length. Above that size of crab, the only types of shells large enough (for predator protection) that are even slightly common are Nerita peloronta (bleeding tooth nerite) and Cittarium pica. $N$. peloronta shells are particularily poor shells for hermit crabs because of the tooth-like projections in the aperture. Thus, increase in (male) size is advantageous up to a point. Beyond that point (in size), there is a good chance that reproductive fitness may drop as a result of a shift in the types of resources available.

A possible solution to this situation may be the adjustments to growth that inhabitation of shells of less-than-desired size can induced. Mark- 
ham (1968) has shown that occupation of sub-optimal shells can not only slow the rate of increase in size of hermit crabs but animals may molt and be smaller after the molt when inhabiting very suboptimal shells. When the supply of large, appropriate kinds of shells is very limited and when no crab will exchange for a shell of the "wrong" kind, de-growth may be the only option available for male hermit crabs.

The non-linearity of the relationship between male size and reproductive opportunities could suggest the idea of alternative reproductive strategies (Austad 1984) in male hermit crabs. Variability in reproductive patterns has been described for other crustaceans (Christy 1987) as well as a variety of other animals. Whether the existence of variation in behavior patterns should lead to the evokation of "alternate strategies" or simply recognize that animals respond to complex situations in complex ways is a matter of some debate (Dunbar 1982). Even though behavioral ecologists may formulate strategies as a means of categorizing the variability in behavior patterns we observe, we must guard against the idea that animals are actually following sets of strategic rules in responding to stimulus inputs and motivational levels.

The suggestion from our data that particular pairs of individuals repeatedly interact is surprising. It is possible that the repeated pairing is a result of nothing more than some degree of sizepairing and the sexually most active individuals finding each other. Alternatively, males could be attempting to make sure that a particular female's eggs are fertilized by their sperm. Because the time of actual fertilization is not known for these crabs, the possible importance of being either the first or last male to copulate is an open question.

Acknowledgements. This is contribution number 435 from the Diseovery Bay Marine Laboratory. This work was supported by grant BNS-8604092 from the Psychobiology Program of the National Science Foundations. We wish to thank the staff at the Discovery Bay Marine Laboratory for their assistance and Catherine Bach for her comments on the manuscript.

\section{References}

Arcese P (1987) Age, instrusion pressure and defence against floaters by territorial male song sparrows. Anim Behav 35:773-784

Arnold SJ (1983) Sexual selection: the interface of theory and empiricism. In: Bateson P (ed) Mate choice. Cambridge University Press, Cambridge, pp 67-107
Austad SN (1984) A classification of alternative reproductive behaviours and methods for field-testing ESS models. Am Zool 24:309-319

Bach C, Hazlett B, Rittschof D (1976) Effects of interspecific competition on fitness of the hermit crab Clibanarius tricolor. Ecology 57:579-586

Bertness MD (1981 a) Competitive dynamics of a tropical hermit crab assemblage. Ecology 62:751-761

Bertness MD (1981 b) The influence of shell-type of hermit crab growth rate and clutch size (Decapoda, Anomura). Crustaceana (Leiden) 40:197-205

Borgia G, Kaatz IM, Condit R (1987) Flower choice and bower decoration in the satin bowerbird Ptilonorhynchus violaceus: a test of hypotheses for the evolution of male display. Anim Behav 35:1129-1139

Christy JH (1987) Female choice and the breeding behavior of the fiddler crab Uca beebei. J Crust Biol 7:624 635

Dunbar RIM (1982) Intraspecific variations in mating strategy. In: Bateson PPG, Klopfer PH (eds) Perspectives in Ethology, vol 5. Plenum Press, New York, pp 385-431

Fotheringham N (1976) Effects of shell stress on the growth of hermit crabs. J Exp Mar Biol Ecol 23:299-305

Fotheringham $N$ (1980) Effects of shell utilization on reproductive patterns in tropical hermit crabs. Mar Biol 55:287-293

Halliday TH (1978) Sexual selection and mate choice. In: Krebs JR, Davies NB (eds) Behavioural ecology an evolutionary approach. Sinauer, Sunderland, pp 180-213

Hazlett BA (1966) Social behavior of the Paguridae and Diogenidae of Curacao. Stud Fauna Curacao 23:1-143

Hazlett BA (1972) Shell fighting and sexual behavior in the hermit crab genera Paguristes and Calcinus, with comments on Pagurus. Bull Mar Sci 22:806-823

Hazlett BA (1981) The behavioral ecology of hermit crabs. Ann Rev Ecol Syst 12:1-22

Hazlett BA (1984) Variations in the pattern of shell exchange among hermit crabs. Anim Behav 32:934-935

Hazlett BA (1987) Hermit crab shell exchange as a model system. Bull Mar Sci 41:99-107

Hazlett BA (1989) Shell exchange in the hermit crab Calcinus tibicen. Anim Behav 37:104-111

Imafuku M (1983) New shell acquisition in the hermit crab, Pagurus geminus. J Ethol 1:91-100

Markham JC (1968) Notes on growth-patterns and shell-utilizations of the hermit crab Pagurus bernhardus (L). Ophelia 5:189-205

McLean RB (1974) Direct shell acquisition by hermit crabs from gastropods. Experientia 30:206-208

Rittschof D (1980) Chemical attraction of hermit crabs and other attendants to simulated gastropod predation sites. J Chem Ecol 6:103-118

Salmon M (1983) Courtship, mating systems and sexual selection in Decapods. In: Rebach S, Dunham DW (eds) Studies in adaptation. The behavior of higher crustacea. Wiley, New York, pp 143-169

Scully EP (1983) The behavioral ecology of competition and resource utilization among hermit crabs. In: Rebach $S$, Dunham DW (eds) Studies in adaptation. The Behavior of higher crustacea. Wiley, New York, pp 23-55

Vance RR (1972) Competition and mechanism of coexistence in three sympatric species of intertidal hermit crabs. Ecology 53:1062-1074 